\title{
EFFECTS OF PARTIAL REPLACEMENT OF FISH OIL WITH DIFFERENT VEGETABLE OILS ON GROWTH, FEED UTILISATION AND FATTY ACID PROFILE OF HYBRID GROUPER JUVENILE (Epinephelus fuscoguttatus $\odot$ E Einephelus lanceolatus $\sigma^{\prime}$ )
}

\begin{abstract}
ANNITA SEOK KIAN YONG*; NUR SUHAILAH SYED MUBARAK and ROSSITA SHAPAWI*
ABSTRACT

This study was done to investigate the effects of fish oil (FO) replacement with different types vegetable oils (VO) on the growth performance, feed utilisation and fatty acid profile of hybrid grouper juvenile (Ephinephelus fuscoguttatus,, ) $x$ giant grouper (Epinephelus lanceolatus, $\mathrm{O}^{1}$ ). Six isoprotein (50\%) and isolipid (12\%) diets were formulated using 100\% FO (as the control) and 50\% of FO was replaced with crude palm oil (CPO), refined, bleached and deodourised (RBD) palm olein (POo), crude palm kernel oil (CPKO), corn oil (CO) and coconut oil (COCO). Triplicate groups of hybrid grouper juvenile (11.12 \pm 0.04 g) were fed with the experimental diets for a period of 12 weeks. Fish fed with RBD POo diet showed significantly higher growth and slightly better feed efficiency than other diets. The different $V O$ affected the body indices, whole body proximate composition and fatty acid profile of the muscle and liver. However, the survival of fish was not affected by the different diets. Present study suggests that RBD POo is the most suitable VO for hybrid grouper.
\end{abstract}

\section{Keywords: hybrid grouper, fish oil, vegetable oil, growth, feed utilisation.}

Date received: 28 May 2018; Sent for revision: 20 June 2018; Received in final form: 5 October 2018; Accepted: 8 January 2019.

\section{INTRODUCTION}

Hybrid grouper is a cross breeding between brownmarbled grouper (Ephinephelus fuscoguttatus, O) $\mathrm{x}$ giant grouper (E. lanceolatus, $\mathrm{O}^{\prime \prime}$ ) which was produced in 2006 at Borneo Marine Research Institute of Universiti Malaysia Sabah (Ch'ng and Senoo, 2008). The hybrid grouper had then become a popular aquaculture fish in Malaysia and other South-east Asian countries. It has high aquaculture demand due to its high egg hatching rate, faster growth, strong tolerance to the environmental factors

\footnotetext{
Borneo Marine Research Institute,

Universiti Malaysia Sabah, Jalan UMS,

88400 Kota Kinabalu, Sabah, Malaysia.

E-mail: annitay@ums.edu.my
}

and resilience to diseases than its parental species (Mustafa et al., 2013; Othman et al., 2015; Koh et al., 2016; Bunlipatanon and U-taynapun, 2017; Arrokhman et al., 2017).

Feed is one of the major factors that plays an important role on the success of the aquaculture sector as it represents $50 \%$ to $70 \%$ of the total production costs (FAO, 2009). In the production of aquaculture feed, fish oil (FO) is the most important lipid sources. It is commonly used as the main lipid source for marine fish feed due to its high digestibility, essential fatty acids (EFA), in particular long chain polyunsaturated fatty acid (PUFA) (Nasopouluo and Zabetakis, 2012) and fat soluble nutrients for normal growth and development of fish (Turchini et al., 2009). However, the rising 
environmental and economic costs of the feedstuff have encouraged its sparing using alternative lipid sources (Olsen et al., 2011; Chen et al., 2017).

Over the past two decades, vegetable oil (VO) has emerged as the alternative source of lipid in aquaculture feed, due to the steadily increasing production, high availability and better economic value of VO (Turchini et al., 2011). However, several studies had reported various results on the use of $\mathrm{VO}$ on the health or growth of freshwater and marine fish species (Glencross et al., 2003; Torstensen et al., 2004; Izquierdo et al., 2005; Mourente and Bell, 2006; Lin and Shiau, 2007; Piedecausa et al., 2007; Shapawi et al., 2008). Among the VO, palm oil is one of the major oils and fats produced and traded in the world today. In 2016, palm oil and palm kernel oil had jointly accounted for $31 \%$ of total output in global production of oils and fats (Oil World, 2017; MPOC, 2017) and Malaysia is the second largest producer of palm oil in the world. Apart from palm oil and palm kernel oil, coconut oil (COCO) is also one of the major oils which is also being locally produced and available in Malaysia and had also contributed to the world oils production (USDA, 2017).

The oil palm fruit produces two types of oils; crude palm oil (CPO) which is derived from the mesocarp and crude palm kernel oil (CPKO) from the kernel. Then the CPO is further processed into refined, bleached and deodourised (RBD) palm olein (POo). All these oils are physically and chemically different form each other and have different fatty acid profiles. $\mathrm{CPO}$ and RBD PO are rich in palmitic acid (C16:0) and oleic acid (C18:1n-9), while CPKO is high in saturated fatty acid, lauric acid (C12:0) and myristic acid (C14:0) (Christine et al., 2014) which shows very similar fatty acid profile with $\mathrm{COCO}$ (Turchini et al., 2010). Besides, COCO is gaining much attention as it is highly digestible (Rosjo et al., 2000) and anti-parasite (Hirazawa et al., 2001).

The uses of VO in aquaculture feed are well documented. In grouper, study on humpback grouper, Cromileptis altivelis (Shapawi et al., 2008) showed that there was no significant difference on growth, survival, feed utilisation, hepatosomatic index and lipid levels in muscle and liver between the fish fed with CPO, RBD PO, soyabean oil and canola oil at $50 \%$ of FO replacement. Besides, better growth performance was also found in brownmarbled grouper, E. fuscoguttatus (Mohd Faudzi, 2013) fed with RBD PO, soyabean oil and canola oil at $50 \%$ of $\mathrm{FO}$ replacement than fish fed with FO. The feed conversion ratio (FCR) was not affected by the experimental treatments.

Various studies also reported on the successful use of corn oil (CO) for several aquaculture species in fish feed (Lin et al., 2007; Lin and Shiau, 2007; Al-Souti et al., 2012; Pereira et al., 2018). At 50\% replacement of $\mathrm{FO}$ with $\mathrm{CO}$, no significant difference in survival, growth performance and hepatosomatic index was found between the FO and $\mathrm{CO}$ treatments in orange spotted grouper, E. coloides (Lin et al., 2007). While on COCO, there is limited study on grouper. Study on the hybrid grouper (E. fuscoguttatus) $\mathrm{x}$ giant grouper (E. lanceolatus) using $\mathrm{COCO}, \mathrm{CO}$ and squid oil showed that $\mathrm{COCO}$ was not the best performing diet compared to fish fed on $\mathrm{CO}$ and squid oil although no significant effect on the growth was observed (Fitriyani et al., 2015).

Therefore, this study was conducted to investigate the suitable type of $\mathrm{VO}$, namely the $\mathrm{CPO}, \mathrm{RBD} \mathrm{POo}, \mathrm{CPKO}, \mathrm{CO}$ and $\mathrm{COCO}$ to partially replace $\mathrm{FO}$ in feed for hybrid grouper juvenile, (E. fuscoguttatus) $\mathrm{x}$ giant grouper (E. lanceolatus).

\section{MATERIALS AND METHOD}

\section{Experimental Diets and Diet Preparation}

Six isoproteic (50\%) and isolipid (12\%) experimental diets were formulated according to the requirement of hybrid grouper juvenile (Mohd Faudzi et al., 2017). Diet with $100 \%$ FO was served as control diet. Other diets were prepared by substituting $50 \%$ of the $\mathrm{FO}$ with different VO; diet with $\mathrm{CPO}, \mathrm{RBD} \mathrm{PO}, \mathrm{CPKO}, \mathrm{CO}$ and $\mathrm{COCO}$ (Table $1)$. The various types of palm oils were obtained from Sawit Kinabalu, Sabah, Malaysia while the $\mathrm{COCO}$ and $\mathrm{CO}$ were purchased from local market.

The experimental diets were prepared by mixing all the ingredients until a homogenous mixture was obtained, then $40 \%$ water was added to form a moist dough before passing the dough through a $3 \mathrm{~mm}$ dice kitchen mincer. The resultant strands of feed were dried in an oven at $40^{\circ} \mathrm{C}$ for 5-6 hr. All the experimental diets were stored in airtight plastic containers and refrigerated at $4^{\circ} \mathrm{C}$ until used. All the diets were subjected to proximate analysis (AOAC, 1999) and fatty acid analysis before the feeding trial.

All diets contained approximately 50\% crude protein and $12 \%$ crude lipid (Table 1 ). The fatty acid composition of $\mathrm{FO}$ and $\mathrm{VO}$ used were different among the different types of oils. FO contains mainly n-3 polyunsaturated fatty acid (PUFA) C20:5n-3 (EPA) and C22:6n-3 (DHA), CPO and RBD POo contains mainly $\mathrm{C} 16: 0$ and $\mathrm{C} 18: 1 \mathrm{n}-9, \mathrm{CPKO}$ contains mainly C12:0, C14:0 and C18:1n-9, CO contains mainly of C18:1n-9 and C18:2n-6 while COCO is abundant with the monosaturated fatty acid C12:0 (Table 2). The fatty acid profile of the oil was well reflected in the fatty acid profiles in the experimental diets (Table 3). The PUFA (C20:4n-6 - C22:6n-3) were detected in all of the $\mathrm{VO}$ experimental diets due to the use of FM and $50 \%$ FO in each diet. 
TABLE 1. INGREDIENT COMPOSITION OF THE EXPERIMENTAL DIET AND PROXIMATE COMPOSITION (\% of dry matter)

\begin{tabular}{|c|c|c|c|c|c|c|}
\hline \multirow{2}{*}{ Ingredients } & \multicolumn{6}{|c|}{ Experimental diet } \\
\hline & FO & $\mathrm{CPO}$ & RBD POo & CPKO & $\mathrm{CO}$ & $\mathrm{COCO}$ \\
\hline a Fish meal & 65.98 & 65.98 & 65.98 & 65.98 & 65.98 & 65.98 \\
\hline b Tapioca starch & 14.75 & 14.75 & 14.75 & 14.75 & 14.75 & 14.75 \\
\hline Alfa- cellulose & 4.50 & 4.50 & 4.50 & 4.50 & 4.50 & 4.50 \\
\hline${ }^{\mathrm{c}}$ Carboxymethyl cellulose (CMC) & 1.50 & 1.50 & 1.50 & 1.50 & 1.50 & 1.50 \\
\hline${ }^{\mathrm{d}}$ Vitamin premix & 3.00 & 3.00 & 3.00 & 3.00 & 3.00 & 3.00 \\
\hline e Mineral premix & 2.00 & 2.00 & 2.00 & 2.00 & 2.00 & 2.00 \\
\hline${ }^{\mathrm{f}}$ Dicalcium phosphate & 1.00 & 1.00 & 1.00 & 1.00 & 1.00 & 1.00 \\
\hline${ }^{g}$ Chromic oxide & 0.50 & 0.50 & 0.50 & 0.50 & 0.50 & 0.50 \\
\hline Fish oil (FO) & 6.77 & 0.77 & 0.77 & 0.77 & 0.77 & 0.77 \\
\hline${ }^{\text {h}}$ Crude palm oil (CPO) & - & 6.00 & - & - & - & - \\
\hline \multicolumn{7}{|l|}{ 'Refined, bleached and deodourised } \\
\hline palm olein (RBD POo) & - & - & 6.00 & - & - & - \\
\hline ¡Crude palm kernel oil (CPKO) & - & - & - & 6.00 & - & - \\
\hline${ }^{\mathrm{k}}$ Corn oil $(\mathrm{CO})$ & - & - & - & - & 6.00 & - \\
\hline \multirow[t]{2}{*}{${ }^{1}$ Coconut oil $(\mathrm{COCO})$} & - & - & - & - & - & 6.00 \\
\hline & \multicolumn{6}{|c|}{ Proximate composition ( $\%$ of dry matter basis) } \\
\hline Moisture & 10.09 & 11.45 & 10.02 & 10.28 & 10.17 & 10.33 \\
\hline Crude protein & 52.94 & 52.99 & 51.62 & 51.91 & 51.76 & 51.65 \\
\hline Crude lipid & 12.18 & 12.75 & 12.36 & 12.87 & 12.14 & 12.36 \\
\hline Crude ash & 15.76 & 15.89 & 15.78 & 15.77 & 15.80 & 15.85 \\
\hline
\end{tabular}

Note: ${ }^{a}$ Fish meal - Danish fish meal, Denmark (75.78\% crude protein). ${ }^{\text {b} T a p i o c a ~ s t a r c h ~-~ T a p i o c a ~ A A A ~ b r a n d, ~ B a k e ~ W i t h ~ M e ~ S d n ~ B h d . ~}$ ${ }^{c}$ Carboxymethyl cellulose (CMC) - Sigma. ${ }^{\mathrm{d}}$ Vitamin premix - ( $\mathrm{g} \mathrm{kg}^{-1}$ premix): ascorbic acid, 45.0; Inosito, 5.0; choline chloride, 75.0; niacin, 4.5; riboflavin, 1.0; pyridoxide $\mathrm{HCl}, 1.0$; thiamine $\mathrm{HCl}, 0.92$; D - calcium panothenate, 3.0; retinyl acetate, 0.60. Vitamin $\mathrm{D} 3$, 0.083; menadione, 1.67; DL alpha tocopherol acetate, 8.0; D - biotin, 0.02; folic acid, 0.09; Vitamin B12, 0.00135. All ingredients were diluted with alpha cellulose to $1 \mathrm{~kg}$. ${ }^{\mathrm{e}}$ Mineral premix - $\left(\mathrm{g} \mathrm{kg}^{-1}\right.$ premix): calcium phosphate monobasic, 270.98; calcium lactate, 327.0; ferrous sulphate, 25.0; magnesium sulphate, 132.0; potassium chloride, 50.0; sodium chloride, 60.0; potassium iodide, 0.15; copper sulphate, 0.785; manganese oxide, 0.8 ; cobalt carbonate, 1.0 ; zinc oxide, 3.0; sodium salenite, 0.011 ; calcium carbonate, 129.274 . ${ }^{\mathrm{f}}$ Dicalcium phosphate R\&M chemical. ${ }^{g}$ Chromic oxide - Sigma. ${ }^{\mathrm{h} C}$ rude palm oil (CPO) - supplied by Sawit Kinabalu. ${ }^{\mathrm{I}}$ Refined, bleached and deodourised palm olein (RPOo) - supplied by Sawit Kinabalu. ${ }^{\mathrm{C} C r u d e}$ palm kernel oil (CPKO) - supplied by Sawit Kinabalu. ${ }^{\mathrm{k}} \mathrm{Corn}$ oil (CO) - cooking oil. ${ }^{1}$ Coconut oil (COCO) - cooking oil.

\section{Feeding trial}

The feeding trial was conducted at the Fish Hatchery, Borneo Marine Research Institute of Universiti Malaysia Sabah, Malaysia. The fish was obtained from a local fish farm at Tawau, Sabah, Malaysia. The fish were acclimatised to experimental condition for a week while feeding with commercial diet (Leong Hup Feedmill, Malaysia; crude protein $44 \%$, crude lipid $8 \%$ ) before the feeding trial. Triplicate groups of hybrid grouper juvenile of initial body weight (BW) $11.12 \pm 0.04 \mathrm{~g}$ were each stocked into 100 litres fibre glass tank at 20 individuals/tank for each dietary treatment. All of the tanks were connected to a flow through water system with water flow rate at 5 litres $\mathrm{min}^{-1}$. The tanks were placed under roofed hatchery which was exposed to natural photoperiods of almost 12 hr light and $12 \mathrm{hr}$ dark. During the feeding trial, the fish were hand-fed up to apparent satiation twice daily at $0800 \mathrm{hr}$ and $1500 \mathrm{hr}$, for a total of 12 weeks. The daily feed intake was recorded to estimate the feed utilisation.
The BW and total length (TL) of fish were measured individually at the beginning and end of the feeding trial, whereas bulk BW was weighed biweekly. Prior to every measurement, the fish were starved for $24 \mathrm{hr}$ to ensure that there was no food in the digestive tract. A commercial anaesthetic (Transmore, NIKA brand) was used to anaesthetise the fish before taking the weight and length measurements to minimise the handling stress. At the end of feeding trials, fish from each treatment were sacrificed for biochemical analysis and estimation of body indices.

\section{Biochemical Analysis}

Proximate composition analyses. Experimental diet and fish whole body proximate composition analyses were done according to the standard method AOAC (1999). The moisture content was determined by drying the sample in oven at $105^{\circ} \mathrm{C}$ for $24 \mathrm{hr}$; ash content was determined by incinerating the dry sample in muffle furnace at $550^{\circ} \mathrm{C}$ for $5 \mathrm{hr}$. Crude protein (Nx6.25) was determined by digesting 
TABLE 2. FATTY ACID COMPOSITIONS (\% of total fatty acids) OF DIFFERENT LIPID SOURCES

\begin{tabular}{|c|c|c|c|c|c|c|}
\hline \multirow{2}{*}{ Fatty acids } & \multicolumn{6}{|c|}{ Type of lipid source } \\
\hline & FO & $\mathrm{CPO}$ & RBD POo & СРКО & $\mathrm{CO}$ & $\mathrm{COCO}$ \\
\hline C 8:0 & - & - & - & 3.28 & - & 6.13 \\
\hline C10:0 & - & - & - & 2.62 & - & 5.12 \\
\hline C12:0 & 0.13 & 0.12 & 0.14 & 48.77 & - & 51.11 \\
\hline C14:0 & 7.69 & 0.83 & 0.79 & 15.54 & 0.03 & 18.66 \\
\hline C16:0 & 22.80 & 44.63 & 42.33 & 8.01 & 10.78 & 8.74 \\
\hline C16:1 & 7.41 & 0.13 & 0.14 & 0.03 & 0.07 & 0.01 \\
\hline C18:0 & 3.38 & 3.59 & 3.84 & 1.91 & 1.63 & 2.73 \\
\hline C18:1n-9 & 15.20 & 41.13 & 43.04 & 17.39 & 28.58 & 5.61 \\
\hline C18:2n-6 & 1.05 & 8.75 & 9.35 & 2.53 & 57.31 & 1.31 \\
\hline C18:3n-3 & 0.80 & 0.21 & 0.12 & - & 0.77 & - \\
\hline C20:0 & 0.32 & 0.28 & 0.28 & 0.09 & 0.32 & 0.07 \\
\hline C20:1 & 6.69 & 0.11 & 0.11 & 0.08 & 0.18 & 0.03 \\
\hline C20:4n-6 & 1.23 & - & - & - & - & - \\
\hline C20:5n-3 & 17.75 & - & - & - & - & - \\
\hline C22:1n-9 & 3.41 & - & - & - & - & - \\
\hline$C 22: 6 n-3$ & 9.19 & - & - & - & - & - \\
\hline Total saturates & 35.07 & 49.45 & 47.38 & 80.22 & 12.76 & 92.56 \\
\hline Total monoenes & 32.71 & 41.37 & 43.29 & 17.50 & 28.83 & 5.65 \\
\hline${ }^{1}$ Total PUFA & 30.08 & 8.96 & 9.47 & 2.53 & 58.08 & 1.31 \\
\hline Total n-3 & 27.80 & 0.21 & 0.12 & 0.00 & 0.77 & 0.00 \\
\hline Total n-6 & 2.28 & 8.75 & 9.35 & 2.53 & 57.31 & 1.31 \\
\hline$n-3 / n-6$ & 12.20 & 0.03 & 0.02 & 0.00 & 0.02 & 0.00 \\
\hline
\end{tabular}

Note: Minor fatty acids (<1\%) not listed was: C4:0, C6:0, C11:0 C13:0, C14:1, C15:0, C17:0, C18:2n9, C18:3n-6, C20:3n6, C22:0, C23:0, C24:0, C24:1. ${ }^{1}$ Total PUFA (polyunsaturated fatty acid) - sum of total n-3 fatty acids and total n-6 fatty acids.

FO - fish oil.

CPO - crude palm oil.

RBD POo - refined, bleached and deodourised palm olein.

CPKO - crude palm kernel oil.

$\mathrm{CO} \quad-$ corn oil.

COCO - coconut oil.

the sample in concentrated sulphuric acid with two tablet of catalyst (Kjeltabs, FOSS Tecator, Sweden) following Kjeldahl method using automatic system model Kjeltec ${ }^{\mathrm{TM}}$ 2300, Foss, Germany. Crude lipid was extracted using petroleum benzene (boiling point $40^{\circ} \mathrm{C}-60^{\circ} \mathrm{C}$ ) with Soxtec $^{\mathrm{TM}} 2043$, Foss, Germany.

Fatty acid analysis. Fatty acid analysis was performed on the $\mathrm{FO}, \mathrm{VO}$, experimental diets, muscle and liver of the fish after the feeding trial. The crude lipid was extracted using chloroform: methanol $(2: 1, \mathrm{v} / \mathrm{v})$ following Folch et al. (1957) and fatty acid methyl esters (FAME) was obtained after the trans-methylation process using sodium hydroxide in methanol (NaOH-methanol) and hydrogen chloride in methanol (HCl-methanol) (Yoshikara and Satoh, 1989). The FAME was analysed using gas chromatography (Shimadzu GC-2010, Shimadzu Corporation, Kyoto, Japan) with helium as carrier gas and nitrogen $\left(\mathrm{N}_{2}\right)$ as make up gas. The FAME was separated using a capillary column $(60 \mathrm{~m} \mathrm{x}$ 0.25 mm ID; BPX70 column, SGE Australia Pty Ltd, Ringwood, Victoria, Australia). The temperature programme ranged from $80^{\circ} \mathrm{C}-230^{\circ} \mathrm{C}\left(4^{\circ} \mathrm{C} \mathrm{min}^{-1}\right.$ from $80^{\circ} \mathrm{C}$ to $180^{\circ} \mathrm{C}$ and $2^{\circ} \mathrm{C} \mathrm{min}^{-1}$ from $181^{\circ} \mathrm{C}$ to $230^{\circ} \mathrm{C}$ ). The peaks obtained were identified by comparing the retention times of each peak with known standards (Supelco ${ }^{\mathrm{TM}} 37$ Component FAME mix, Supleco Inc. Bellefonte, USA).

\section{Statistical Analysis}

Data on growth performance, survival, feed utilisation, body indices and body composition of hybrid grouper juveniles were analysed using one way analysis of variance (ANOVA) in the Statistical Package for the Social Science, SPSS 20.0. Tukey's multiple range tests were used to determine significant differences between all of the treatment means at $P$ values is $<0.05$.

\section{RESULTS}

\section{Growth Performance, Survival and Feed Utilisation}

At the end of the feeding trial, fish fed with RBD POo achieved the highest growth and it was significantly higher $(\mathrm{P}<0.05)$ than fish fed with other diets in terms of weight gain (WG) and specific 
TABLE 3. FATTY ACID COMPOSITIONS (\% of total fatty acids) OF EXPERIMENTAL DIETS

\begin{tabular}{|c|c|c|c|c|c|c|}
\hline \multirow{2}{*}{ Fatty acids } & \multicolumn{6}{|c|}{ Experimental diets } \\
\hline & FO & $\mathrm{CPO}$ & RBD POo & СРКО & $\mathrm{CO}$ & $\mathrm{COCO}$ \\
\hline $\mathrm{C} 12: 0$ & 0.12 & 0.10 & 0.12 & 26.81 & 0.04 & 26.48 \\
\hline C14:0 & 7.08 & 3.60 & 3.67 & 12.73 & 3.25 & 15.15 \\
\hline C14:1 & 0.22 & 0.12 & 0.12 & 0.14 & 0.13 & 0.13 \\
\hline C15:0 & 0.44 & 0.22 & 0.23 & 0.25 & 0.22 & 0.24 \\
\hline C16:0 & 19.89 & 34.95 & 33.44 & 14.50 & 15.74 & 16.73 \\
\hline C16:1 & 6.28 & 2.35 & 2.64 & 2.52 & 2.36 & 2.69 \\
\hline C17:0 & 0.32 & 0.18 & 0.19 & 0.15 & 0.17 & 0.15 \\
\hline C18:0 & 2.94 & 3.44 & 3.47 & 2.58 & 2.33 & 3.26 \\
\hline C18:1n-9 & 13.88 & 31.16 & 32.76 & 16.11 & 23.64 & 10.83 \\
\hline C18:2n-6 & 2.11 & 6.44 & 6.57 & 2.71 & 34.28 & 2.65 \\
\hline C18:3n-3 & 1.02 & 0.67 & 0.57 & 0.58 & 1.04 & 0.57 \\
\hline C20:0 & 0.29 & 0.31 & 0.29 & 0.18 & 0.33 & 0.17 \\
\hline C20:1 & 9.09 & 5.40 & 5.24 & 5.86 & 5.47 & 4.97 \\
\hline$C 20: 4 n-6$ & 0.99 & 0.31 & 0.31 & 0.32 & 0.30 & 0.33 \\
\hline$C 20: 5 n-3$ & 13.04 & 1.50 & 1.06 & 1.50 & 0.88 & 1.58 \\
\hline C22:1n-9 & 9.34 & 1.08 & 1.00 & 0.07 & 0.07 & 0.16 \\
\hline$C 22: 6 n-3$ & 9.82 & 5.20 & 5.05 & 5.59 & 5.20 & 5.97 \\
\hline Total saturates & 31.09 & 42.80 & 41.42 & 60.06 & 22.03 & 66.39 \\
\hline Total monoenes & 38.81 & 40.11 & 41.76 & 24.70 & 31.67 & 18.78 \\
\hline${ }^{1}$ Total PUFA & 26.97 & 32.82 & 32.77 & 22.74 & 45.14 & 21.70 \\
\hline Total n-3 & 23.88 & 7.37 & 6.68 & 7.69 & 7.12 & 8.12 \\
\hline Total n-6 & 3.10 & 6.75 & 6.88 & 3.03 & 34.58 & 2.98 \\
\hline$n-3 / n-6$ & 7.70 & 1.09 & 0.97 & 2.54 & 0.21 & 2.72 \\
\hline
\end{tabular}

Note: Minor fatty acids (<1\%) not listed was: C4:0, C11:0, C13:0, C18:2n9, C18:3n-6, C20:3n6,C22:0, C23:0, C24:0, C24:1. ${ }^{1}$ Total PUFA (polyunsaturated fatty acid) - sum of total n-3 fatty acids and total n- 6 fatty acids.

FO - fish oil.

CPO - crude palm oil.

RBD POo - refined, bleached and deodourised palm olein.

CPKO - crude palm kernel oil.

$\mathrm{CO}$ - corn oil.

COCO - coconut oil.

growth rate (SGR) (Table 4). On the other hand, the lowest growth was observed in fish fed with $\mathrm{CO}$ diet. Besides, the growth performance of fish fed with $\mathrm{FO}, \mathrm{CPO}, \mathrm{CPKO}, \mathrm{CO}$ and $\mathrm{COCO}$ diets showed no significant differences among the treatments. The experimental diets did not influence the survival of the fish as high survival rate of $98.81 \%-100 \%$ was observed among the treatments.

For feed utilisation, significantly higher total feed intake was found in fish fed with RBD POo diet compared to fish fed with $\mathrm{CO}$ diet $(\mathrm{P}<0.05)$. No significant difference was found among fish fed with $\mathrm{CPO}, \mathrm{CPKO}, \mathrm{CO}$ and $\mathrm{COCO}$ diets with $\mathrm{FO}$ diet. For food conversion ratio (FCR), no significant effect was found on fish fed the different experimental diets. However, overall, the fish fed with FO, CPO, RBD POo and CO demonstrated better FCR in the range of 1.26-1.34, while fish fed with CPKO was slightly higher at 1.47 and the highest was in the fish fed with $\mathrm{COCO}$ at 1.62. The protein efficiency ratio (PER) was not significantly different among the treatments. However, higher efficiency was found in fish fed with FO, CPO and RBD POo. Similarly, no significant differences were observed on the net protein utilisation (NPU), although fish fed with
RBD POo and CO showed higher NPU than other diets.

\section{Body Indices and Whole Body Proximate Composition}

Results on the body indices and the whole body proximate composition of hybrid grouper juvenile at theend of the feeding trial are presented in Table 5. The condition factor $(\mathrm{CF})$ was not significantly different among the treatments $(\mathrm{P}>0.05)$. The hepato-somatic index (HSI) in fish fed with FO, CPO and RBD POo was significantly higher than fish fed with CPKO and $\mathrm{COCO}$. On the other hand, the viscerosomatic index (VSI) was significantly higher in fish fed with $\mathrm{CO}$ and $\mathrm{COCO}$ compared to fish fed with $\mathrm{CPKO}$ while no significant difference was observed among other treatments. For intraperitoneal fat (IPF), an almost similar pattern with HSI was observed.

The whole body proximate composition showed that the body protein of fish fed with $\mathrm{CPO}$ and RBD POo was almost similar with the fish fed with FO without significant difference $(P>0.05)$. While significantly higher body protein content was observed in fish fed with $\mathrm{CPKO}, \mathrm{CO}$ and $\mathrm{COCO}$ 
TABLE 4. GROWTH PERFORMANCES, SURVIVAL AND FEED UTILISATION OF HYBRID GROUPER JUVENILE IN 12 WEEKS OF FEEDING TRIAL

\begin{tabular}{|c|c|c|c|c|c|c|}
\hline \multirow{2}{*}{ Variable } & \multicolumn{6}{|c|}{ Experimental diet } \\
\hline & FO & $\mathrm{CPO}$ & RBD POo & СРКО & $\mathrm{CO}$ & $\mathrm{COCO}$ \\
\hline${ }^{1} \mathrm{IBW}$ (g/fish) & $11.13 \pm 0.88$ & $11.15 \pm 0.03$ & $11.15 \pm 0.04$ & $11.15 \pm 0.11$ & $11.12 \pm 0.06$ & $11.12 \pm 0.1$ \\
\hline${ }^{2} \mathrm{FBW}$ (g/fish) & $121.93 \pm 2.82^{\mathrm{a}}$ & $122.34 \pm 3.60^{\mathrm{a}}$ & $148.57 \pm 9.0^{\mathrm{b}}$ & $122.16 \pm 1.24^{\mathrm{a}}$ & $109.95 \pm 4.23^{\mathrm{a}}$ & $121.15 \pm 4.48^{\mathrm{a}}$ \\
\hline${ }^{3} \mathrm{WG}(\%)$ & $995.76 \pm 2.85^{\mathrm{a}}$ & $997.13 \pm 3.61^{\mathrm{a}}$ & $1232.75 \pm 9.02^{\mathrm{b}}$ & $995.58 \pm 1.18^{\mathrm{a}}$ & $888.82 \pm 4.22^{\mathrm{a}}$ & $989.65 \pm 8.9^{\mathrm{a}}$ \\
\hline${ }^{4}$ SGR $(\%)$ & $4.05 \pm 1.17^{\mathrm{a}}$ & $4.12 \pm 1.20^{\mathrm{a}}$ & $4.32 \pm 1.29^{b}$ & $4.17 \pm 1.25 \mathrm{a}$ & $3.99 \pm 1.21^{\mathrm{a}}$ & $4.00 \pm 1.14^{\mathrm{a}}$ \\
\hline Survival $(\%)$ & $99.52 \pm 0.81$ & 100.00 & $99.05 \pm 2.52$ & $98.81 \pm 2.50$ & $99.52 \pm 0.82$ & $98.81 \pm 2.50$ \\
\hline${ }^{5} \mathrm{FI}$ (g/fish) & $153.52 \pm 2.70^{\mathrm{a}}$ & $162.44 \pm 2.37^{\mathrm{ab}}$ & $176.06 \pm 5.31^{\mathrm{b}}$ & $145.75 \pm 5.31^{\mathrm{ab}}$ & $132.38 \pm 2.76^{\mathrm{a}}$ & $156.39 \pm 5.67^{\mathrm{ab}}$ \\
\hline${ }^{6} \mathrm{FCR}$ & $1.26 \pm 0.07$ & $1.32 \pm 0.16$ & $1.29 \pm 0.11$ & $1.47 \pm 0.14$ & $1.34 \pm 0.08$ & $1.62 \pm 0.43$ \\
\hline${ }^{7} \mathrm{PER}$ & $1.36 \pm 0.80$ & $1.32 \pm 0.15$ & $1.36 \pm 0.12$ & $1.19 \pm 0.11$ & 1.250 .07 & $1.20 \pm 0.14$ \\
\hline${ }^{8} \mathrm{NPU}$ & $22.75 \pm 1.38$ & $23.12 \pm 2.54$ & $24.28 \pm 2.08$ & $21.90 \pm 2.05$ & $26.67 \pm 1.47$ & $23.22 \pm 2.68$ \\
\hline
\end{tabular}

Note: Mean \pm standard deviation $(\mathrm{n}=3)$; within the same row with different letters are significantly different at $\mathrm{P}<0.05$. ${ }^{1} \mathrm{IBW}$ - initial body weight. ${ }^{2} \mathrm{FBW}$ - final body weight. ${ }^{3} \mathrm{WG}$ - weight gain $=100 \times[$ final body weight $(\mathrm{g})$ - initial body weight $(\mathrm{g})] /$ initial body weight $(\mathrm{g})$. ${ }^{4} \mathrm{SGR}$ - specific growth rate $=100 \times[$ Ln final body weight $(\mathrm{g})$ - Ln initial body weight $(\mathrm{g})] /\left(\right.$ days of feeding trial). ${ }^{5} \mathrm{FI}-$ feed intake $=($ total feed intake (g)/number of fishes) in 12 weeks of feeding trial. ${ }^{6} \mathrm{FCR}$ - feed conversion ratio = dry feed consumed $(\mathrm{g}) /$ wet weight gain $(\mathrm{g})$. ${ }^{7}$ PER - protein efficiency ratio $=$ wet weight gain $(\mathrm{g}) /$ total protein intake $(\mathrm{g}) .{ }^{8} \mathrm{NPU}-$ net protein utilisation $=100 \times($ final - initial fish body protein)/total protein intake.

FO - fish oil.

$\mathrm{CPO} \quad$ - crude palm oil.

RBD POo - refined, bleached and deodourised palm olein.

CPKO - crude palm kernel oil.

$\mathrm{CO} \quad-$ corn oil.

COCO - coconut oil.

TABLE 5. BODY INDICES AND WHOLE BODY PROXIMATE COMPOSITION OF HYBRID GROUPER JUVENILE AT THE END OF THE 12 WEEKS OF FEEDING TRIAL

\begin{tabular}{|c|c|c|c|c|c|c|}
\hline \multirow{2}{*}{ Variable } & \multicolumn{6}{|c|}{ Experimental feed } \\
\hline & FO & $\mathrm{CPO}$ & RBD POo & СРКО & $\mathrm{CO}$ & $\mathrm{COCO}$ \\
\hline${ }^{1} \mathrm{CF}$ & $3.16 \pm 0.94$ & $3.09 \pm 0.28$ & $3.35 \pm 0.44$ & $3.04 \pm 0.13$ & $2.99 \pm 0.25$ & $2.93 \pm 0.22$ \\
\hline${ }^{2} \mathrm{HSI}(\%)$ & $1.38 \pm 0.28^{\mathrm{b}}$ & $1.51 \pm 0.18^{\mathrm{b}}$ & $1.52 \pm 0.08^{\mathrm{b}}$ & $1.15 \pm 0.19^{a}$ & $1.31 \pm 0.20^{\mathrm{ab}}$ & $1.16 \pm 0.07^{\mathrm{a}}$ \\
\hline${ }^{3}$ VSI $(\%)$ & $10.31 \pm 0.3^{\mathrm{ab}}$ & $10.39 \pm 0.60^{\mathrm{ab}}$ & $10.40 \pm 0.67^{\mathrm{ab}}$ & $9.23 \pm 1.32^{\mathrm{a}}$ & $11.31 \pm 0.86^{b}$ & $11.59 \pm 1.19^{b}$ \\
\hline${ }^{4} \mathrm{IPF}(\%)$ & $2.44 \pm 0.16^{\mathrm{ab}}$ & $2.50 \pm 0.30^{\mathrm{ab}}$ & $2.88 \pm 0.29^{\mathrm{b}}$ & $2.32 \pm 0.60^{\mathrm{ab}}$ & $2.10 \pm 0.10^{\mathrm{a}}$ & $2.34 \pm 0.12^{\mathrm{ab}}$ \\
\hline Moisture & $70.68 \pm 0.39$ & $68.14 \pm 1.34$ & $70.28 \pm 0.10$ & $69.23 \pm 1.72$ & $70.72 \pm 1.65$ & $69.86 \pm 0.63$ \\
\hline Protein & $16.42 \pm 0.6^{\mathrm{a}}$ & $16.99 \pm 0.40^{\mathrm{a}}$ & $17.40 \pm 0.22^{\mathrm{a}}$ & $18.66 \pm 0.4 b$ & $18.44 \pm 0.1^{\mathrm{b}}$ & $18.48 \pm 0.01^{b}$ \\
\hline Whole body lipid & $4.43 \pm 0.16^{\mathrm{a}}$ & $4.54 \pm 0.29^{a}$ & $4.47 \pm 0.47^{\mathrm{a}}$ & $4.44 \pm 0.10^{\mathrm{a}}$ & $5.28 \pm 0.08^{\mathrm{b}}$ & $5.42 \pm 0.20^{b}$ \\
\hline Ash & $5.68 \pm 0.10$ & $5.98 \pm 0.29$ & $5.77 \pm 0.02$ & $6.37 \pm 0.73$ & $5.56 \pm 0.22$ & $5.89 \pm 0.20$ \\
\hline Liver lipid (dry weight) & $25.29 \pm 1.2^{\mathrm{b}}$ & $29.88 \pm 1.33^{c}$ & $30.67 \pm 0.75^{c}$ & $21.37 \pm 0.51^{\mathrm{a}}$ & $26.42 \pm 0.4^{\mathrm{b}}$ & $21.31 \pm 1.05^{\mathrm{a}}$ \\
\hline Muscle lipid (dry weight) & $7.62 \pm 1.82^{\mathrm{a}}$ & $7.83 \pm 1.06^{\mathrm{a}}$ & $7.93 \pm 0.57^{\mathrm{ab}}$ & $6.59 \pm 0.84^{\mathrm{a}}$ & $9.20 \pm 1.19^{\mathrm{ab}}$ & $10.91 \pm 0.72^{b}$ \\
\hline
\end{tabular}

Note: Mean \pm standard deviation $(\mathrm{n}=3)$; within the same row with different letters are significantly different at $\mathrm{P}<0.05$. ${ }^{1} \mathrm{CF}-\mathrm{condition}$ factor $=\left[\right.$ fish body weight $\left.(\mathrm{g}) /(\text { total length }(\mathrm{cm}))^{3}\right] \times 100 .{ }^{2} \mathrm{HSI}-$ hepatosomatic index $=[$ liver weight $(\mathrm{g}) /$ fish body weight $(\mathrm{g})] \times 100,{ }^{3} \mathrm{VSI}-$ viscerosomatic index $=[$ viscera organ weight $(\mathrm{g}) /$ fish body weight $(\mathrm{g})] \times 100,{ }^{4} \mathrm{IPF}$ - intraperitoneal fat $=[$ Intraperitoneal fat weight $(\mathrm{g}) /$ fish body wet weight $(\mathrm{g})] \times 100$.

FO - fish oil.

$\mathrm{CPO} \quad$ - crude palm oil.

RBD POo - refined, bleached and deodourised palm olein.

CPKO - crude palm kernel olein.

$\mathrm{CO} \quad-$ corn oil.

COCO - coconut oil.

than fish fed with FO $(\mathrm{P}<0.05)$. The body lipid content showed that the fish fed with FO, CPO, RBD $\mathrm{PO}$ and $\mathrm{CPKO}$ was significantly lower than the fish fed with $\mathrm{CO}$ and $\mathrm{COCO}$. While the body moisture and ash content was not affected by the treatments.

\section{Fatty Acid Profile}

Generally, the major fatty acid profile of the fish muscle (Table 6) and liver (Table 7) reflected the fatty acid profile of respective experimental diets (Table 3). 
TABLE 6. FATTY ACID COMPOSITIONS (\% of total fatty acids) AND LIPID CONTENT OF FISH MUSCLE TISSUE AT THE END OF THE FEEDING TRIAL

\begin{tabular}{|c|c|c|c|c|c|c|}
\hline \multirow{2}{*}{ Fatty acid } & \multicolumn{6}{|c|}{ Experimental diets } \\
\hline & FO & CPO & RBD POo & СРКО & $\mathrm{CO}$ & $\mathrm{COCO}$ \\
\hline C12:0 & $2.04 \pm 0.50^{\mathrm{b}}$ & - & - & $12.56 \pm 0.15^{c}$ & - & $17.45 \pm 0.30^{\mathrm{d}}$ \\
\hline C14:0 & $7.55 \pm 0.10^{\mathrm{b}}$ & $3.42 \pm 0.07^{\mathrm{a}}$ & $3.22 \pm 0.01^{\mathrm{a}}$ & $13.43 \pm 0.17^{c}$ & $3.56 \pm 0.40^{\mathrm{a}}$ & $15.01 \pm 0.08^{\mathrm{d}}$ \\
\hline C16:0 & $25.58 \pm 0.06^{\mathrm{d}}$ & $34.06 \pm 0.12^{\mathrm{f}}$ & $32.37 \pm 0.08^{e}$ & $18.89 \pm 0.14^{\mathrm{a}}$ & $20.34 \pm 0.34^{\mathrm{b}}$ & $22.10 \pm 0.07^{c}$ \\
\hline C16:1 & $4.57 \pm 0.01^{\mathrm{e}}$ & $2.10 \pm 0.02^{\mathrm{ab}}$ & $2.36 \pm 0.01^{c}$ & $1.98 \pm 0.06^{\mathrm{a}}$ & $2.27 \pm 0.08 b^{c}$ & $2.64 \pm 0.02^{\mathrm{d}}$ \\
\hline C18:0 & $6.00 \pm 0.02^{\mathrm{d}}$ & $6.37 \pm 0.01^{\mathrm{cd}}$ & $5.68 \pm 0.03^{\mathrm{b}}$ & $5.47 \pm 0.09^{\mathrm{b}}$ & $4.69 \pm 0.12^{\mathrm{a}}$ & $6.22 \pm 0.07^{c}$ \\
\hline C18:1n-9 & $16.35 \pm 0.07^{\mathrm{b}}$ & $29.18 \pm 0.07^{e}$ & $30.84 \pm 0.08^{f}$ & $19.60 \pm 0.20^{c}$ & $22.07 \pm 0.41^{\mathrm{d}}$ & $14.35 \pm 0.01^{\mathrm{a}}$ \\
\hline C18:2n-6 & $2.30 \pm 0.01^{\mathrm{a}}$ & $6.28 \pm 0.03^{c}$ & $6.18 \pm 0.01^{c}$ & $3.85 \pm 0.03^{\mathrm{b}}$ & $24.38 \pm 0.26^{\mathrm{d}}$ & $2.44 \pm 0.01^{\mathrm{a}}$ \\
\hline C18:3n-3 & $0.66 \pm 0.07 b^{c}$ & $0.46 \pm 0.02^{\mathrm{ab}}$ & $0.45 \pm 0.01^{\mathrm{a}}$ & $0.77 \pm 0.04^{\mathrm{d}}$ & $0.66 \pm 0.05^{\mathrm{cd}}$ & $0.43 \pm 0.02^{\mathrm{a}}$ \\
\hline C20:1 & $4.32 \pm 0.03^{\mathrm{d}}$ & $3.04 \pm 0.04^{\mathrm{a}}$ & $2.95 \pm 0.01^{\mathrm{a}}$ & $3.97 \pm 0.06^{c}$ & $3.52 \pm 0.04^{\mathrm{b}}$ & $3.94 \pm 0.02^{c}$ \\
\hline C20:4n-6 & $1.29 \pm 0.02^{\mathrm{d}}$ & $0.59 \pm 0.01^{\mathrm{ab}}$ & $0.64 \pm 0.01 b^{c}$ & $0.73 \pm 0.01^{\mathrm{c}}$ & $0.53 \pm 0.04^{\mathrm{a}}$ & $0.59 \pm 0.01^{\mathrm{ab}}$ \\
\hline C20:5n-3 & $7.03 \pm 0.01^{\mathrm{f}}$ & $3.19 \pm 0.010^{\mathrm{b}}$ & $3.94 \pm 0.05^{\mathrm{e}}$ & $3.77 \pm 0.01^{\mathrm{ab}}$ & $2.53 \pm 0.04^{\mathrm{d}}$ & $3.50 \pm 0.01^{\mathrm{c}}$ \\
\hline C22:1n-9 & $0.82 \pm 0.01^{\mathrm{c}}$ & $0.36 \pm 0.01^{\mathrm{a}}$ & $0.34 \pm 0.02^{\mathrm{a}}$ & $0.48 \pm 0.04^{\mathrm{b}}$ & $0.36 \pm 0.03^{\mathrm{a}}$ & $0.54 \pm 0.01^{\mathrm{b}}$ \\
\hline$C 22: 6 n-3$ & $18.45 \pm 0.10^{\mathrm{d}}$ & $9.74 \pm 0.07^{\mathrm{a}}$ & $9.35 \pm 0.06^{\mathrm{a}}$ & $12.20 \pm 0.17^{c}$ & $10.35 \pm 0.24^{\mathrm{b}}$ & $9.40 \pm 0.07^{a}$ \\
\hline Total saturates & $41.17 \pm 0.17$ & $43.85 \pm 0.06$ & $41.27 \pm 0.04$ & $50.35 \pm 0.14$ & $28.59 \pm 0.30$ & $60.78 \pm 0.13$ \\
\hline Total monoenes & $27.40 \pm 0.02$ & $35.42 \pm 0.03$ & $37.17 \pm 0.03$ & $27.91 \pm 0.06$ & $28.93 \pm 0.04$ & $22.20 \pm 0.02$ \\
\hline${ }^{1}$ Total PUFA & $29.35 \pm 0.03$ & $19.65 \pm 0.07$ & $20.49 \pm 0.04$ & $20.72 \pm 0.04$ & $40.36 \pm 0.17$ & $16.04 \pm 0.04$ \\
\hline Total n-3 PUFA & $26.08 \pm 0.07$ & $13.39 \pm 0.23$ & $13.74 \pm 0.08$ & $16.73 \pm 0.05$ & $13.53 \pm 0.45$ & $13.34 \pm 0.10$ \\
\hline Total n-6 PUFA & $3.27 \pm 0.02$ & $6.26 \pm 0.02$ & $6.75 \pm 0.02$ & $3.98 \pm 0.02$ & $26.830 \pm 0.17$ & $2.70 \pm 0.03$ \\
\hline Muscle lipid content (\%) & $7.62 \pm 1.82^{\mathrm{a}}$ & $7.83 \pm 1.06^{\mathrm{a}}$ & $7.93 \pm 0.57^{\mathrm{ab}}$ & $6.59 \pm 0.84^{\mathrm{a}}$ & $9.20 \pm 1.19^{a b}$ & $10.91 \pm 0.72^{\mathrm{b}}$ \\
\hline
\end{tabular}

Note: Minor fatty acids that less than 1\% were not listed. These included C4:0, C13:0, C15:0, C20:0, C22:0, C23:0, C24:0.

${ }^{1}$ Total PUFA (polyunsaturated fatty acid) - sum of total n-3 fatty acids and total n-6 fatty acids.

FO - fish oil.

CPO - crude palm oil.

RBD POo - refined, bleached and deodourised palm olein.

CPKO - crude palm kernel oil.

$\mathrm{CO} \quad-$ corn oil.

COCO - coconut oil.

TABLE 7. FATTY ACID COMPOSITIONS (\% of total fatty acids) OF LIVER TISSUE OF HYBRID GROUPER JUVENILE

\begin{tabular}{|c|c|c|c|c|c|c|}
\hline \multirow{2}{*}{ Fatty acid } & \multicolumn{5}{|c|}{ Experimental diets } & \multirow[b]{2}{*}{$\mathrm{COCO}$} \\
\hline & FO & $\mathrm{CPO}$ & RBD POo & СРКО & $\mathrm{CO}$ & \\
\hline C12:0 & - & - & - & $5.32 \pm 0.06^{\mathrm{a}}$ & - & $9.17 \pm 0.14^{b}$ \\
\hline C14:0 & $9.92 \pm 0.74^{c}$ & $4.60 \pm 0.40^{\mathrm{a}}$ & $5.25 \pm 0.86^{\mathrm{ab}}$ & $17.18 \pm 3.20^{\mathrm{d}}$ & $5.77 \pm 0.43^{\mathrm{ab}}$ & $23.21 \pm 0.35^{\mathrm{d}}$ \\
\hline C16:0 & $39.48 \pm 1.01^{\mathrm{b}}$ & $45.57 \pm 0.26^{c}$ & $45.23 \pm 0.58^{\mathrm{bc}}$ & $30.11 \pm 1.58 b^{c}$ & $33.57 \pm 4.00^{\mathrm{ab}}$ & $31.46 \pm 0.78^{\mathrm{ab}}$ \\
\hline C16:1 & $7.15 \pm 1.15^{\mathrm{b}}$ & $3.02 \pm 0.62^{\mathrm{a}}$ & $3.22 \pm 0.38^{\mathrm{a}}$ & $3.69 \pm 0.17^{a}$ & $3.37 \pm 0.50^{\mathrm{a}}$ & $3.69 \pm 0.17^{\mathrm{a}}$ \\
\hline C18:0 & $7.20 \pm 0.56^{c}$ & $5.30 \pm 0.33^{\mathrm{a}}$ & $5.80 \pm 0.69^{\mathrm{ab}}$ & $6.65 \pm 0.09^{a}$ & $5.26 \pm 0.19^{a}$ & $6.65 \pm 0.07^{\mathrm{bc}}$ \\
\hline C18:1n-9 & $19.27 \pm 1.40^{\mathrm{ab}}$ & $29.67 \pm 0.78 d^{e}$ & $33.12 \pm 0.15^{\mathrm{e}}$ & $15.38 \pm 0.20 b^{c}$ & $25.26 \pm 0.21 \mathrm{~d}^{\mathrm{c}}$ & $15.38 \pm 0.16^{\mathrm{a}}$ \\
\hline C18:2n-6 & $1.16 \pm 0.22^{\mathrm{a}}$ & $2.23 \pm 0.20^{\mathrm{a}}$ & $2.60 \pm 1.23^{\mathrm{a}}$ & $1.03 \pm 0.08^{\mathrm{a}}$ & $12.05 \pm 0.77^{\mathrm{b}}$ & $1.03 \pm 0.08^{\mathrm{a}}$ \\
\hline C20:1 & $5.69 \pm 0.68$ & $4.12 \pm 1.44$ & $4.80 \pm 0.43$ & $5.75 \pm 1.00$ & $5.96 \pm 0.16$ & $5.75 \pm 1.01$ \\
\hline$C 20: 5 n-3$ & $1.79 \pm 0.24^{\mathrm{b}}$ & $0.35 \pm 0.02^{\mathrm{a}}$ & $0.72 \pm 0.05^{\mathrm{a}}$ & $0.31 \pm 0.02^{\mathrm{a}}$ & $0.30 \pm 0.70^{\mathrm{a}}$ & $0.31 \pm 0.02^{\mathrm{a}}$ \\
\hline C22:1n-9 & $0.67 \pm 0.05^{\mathrm{a}}$ & $0.37 \pm 0.02^{\mathrm{a}}$ & $0.35 \pm 0.05^{\mathrm{a}}$ & $0.47 \pm 0.09^{c}$ & $4.80 \pm 0.82^{\mathrm{b}}$ & $0.38 \pm 0.08^{\mathrm{a}}$ \\
\hline$C 22: 6 n-3$ & $14.23 \pm 0.69^{\mathrm{d}}$ & $6.39 \pm 0.22^{b}$ & $7.57 \pm 0.22^{c}$ & $2.87 \pm 0.10^{\mathrm{a}}$ & $3.37 \pm 0.34^{\mathrm{a}}$ & $2.97 \pm 0.06^{\mathrm{a}}$ \\
\hline Total saturates & $56.60 \pm 0.77$ & $55.47 \pm 0.33$ & $56.28 \pm 0.71$ & $59.26 \pm 1.24$ & $44.60 \pm 1.54$ & $61.32 \pm 0.40$ \\
\hline Total monoenes & $32.78 \pm 0.82$ & $37.18 \pm 0.72$ & $41.49 \pm 0.25$ & $25.29 \pm 0.37$ & $39.39 \pm 0.43$ & $25.20 \pm 0.36$ \\
\hline${ }^{1}$ Total PUFA & $18.43 \pm 0.22$ & $10.25 \pm 0.15$ & $12.28 \pm 0.14$ & $4.44 \pm 0.08$ & $18.73 \pm 0.47$ & $4.76 \pm 0.07$ \\
\hline Total n-3 PUFA & $16.02 \pm 0.47$ & $6.75 \pm 0.12$ & $8.29 \pm 0.14$ & $3.18 \pm 0.06$ & $3.67 \pm 0.52$ & $3.28 \pm 0.04$ \\
\hline Total n-6 PUFA & $2.41 \pm 0.15$ & $3.25 \pm 0.31$ & $3.99 \pm 0.25$ & $1.26 \pm 0.08$ & $15.06 \pm 0.77$ & $1.48 \pm 0.08$ \\
\hline Liver lipid content $(\%)$ & $25.29 \pm 1.2^{\mathrm{b}}$ & $29.88 \pm 1.33^{c}$ & $30.67 \pm 0.75^{c}$ & $21.37 \pm 0.51^{\mathrm{a}}$ & $26.42 \pm 0.4^{b}$ & $21.31 \pm 1.05^{\mathrm{a}}$ \\
\hline
\end{tabular}

Note: Minor fatty acids that less than 1\% were not listed. These included: C4:0, C13:0, C17:0, C20:0, C22:0, C23:0, C24:0, ${ }^{1}$ Total PUFA (polyunsaturated fatty acid) - sum of total n-3 fatty acids and total n- 6 fatty acids.

FO - fish oil.

CPO - crude palm oil.

RBD POo - refined, bleached and deodourised palm olein.

CPKO - crude palm kernel oil.

$\mathrm{CO} \quad-$ corn oil.

COCO - coconut oil. 
There were significant differences among all the fatty acids between the diets. Comparison between the fatty acid profile of the muscle and liver showed that in all of the treatments the fish muscle contained higher percentage of PUFA particularly C20:4n6, C20:5n-3(EPA) and C22:6n-3(DHA), while the liver contained higher percentage of saturated (C14:0, C16:0, C18:0) and monosaturated (C16:1, C18:1n-9) fatty acids.

\section{DISCUSSION}

The present study showed that all of the hybrid grouper juvenile responded well to the experimental diets which were $50 \%$ partially replaced with various VO. The different types of VO with different fatty acid profile affected the growth of the fish. Hybrid grouper juvenile fed with $\mathrm{CPO}, \mathrm{CPKO}, \mathrm{CO}$ and COCO showed almost similar growth performance with the control fish fed with FO. Fish fed with RBD POo showed significantly higher body weight gain (WG), and SGR than other diets $(\mathrm{P}<0.05)$. Growth of the hybrid grouper brown-marbled grouper $x$ giant grouper in the present study was comparable to those reported in previous studies (Firdaus et al., 2016; Mohd Faudzi et al., 2017; Ismail et al., 2018). Besides, the survival of the hybrid grouper in the present study was not affected by the diets. Similar results have also been reported in studies on hybrid grouper (Firdaus et al., 2016; Mohd Faudzi et al., 2017; Ismail et al., 2018).

In the present study, hybrid grouper fed with RBD POo showed significantly better growth than fish fed with FO and almost higher feed efficiency compared to FO. This is different with the parental fish, brown-marbled grouper. At similar level of FO replacement with the present study, the brownmarbled grouper fed with RBD POo showed no significant difference on growth compared to fish fed with FO (Mohd Faudzi, 2013). Similarly, the study on humpback grouper, C. altivelis (Shapawi et al., 2008) reported at 50\% replacement level of FO with CPO and RBD POo no significant different on the growth was observed compared to the control fish fed with FO diet. Among the VO tested, RBD POo is the most suitable VO for hybrid grouper. The highest growth performance and feed intake in fish fed with RBD POo showed that the hybrid grouper can utilise the feed well for growth.

In barramundi, Lates calcarifer, a complete replacement of FO with $\mathrm{CPO}$ and $\mathrm{RBD}$ POo in a poultry meal-based diets also demonstrated similar growth performance with fish fed the control diet FO (Wan Ahmad et al., 2013). Positive results on the use of PO products were also documented on freshwater fish (Kamarudin et al., 2012; Ng et al., 2013). Study on Malaysian mahseer, Tor tambroides highest growth performance was found in fish diet replaced with $50 \%$ and $100 \%$ of palm oil (Kamarudin et al., 2012). In a study on red hybrid tilapia, Oreochromis sp., the growth performance and feeding efficiencies in fish fed with crude $\mathrm{PO}$ at $50 \%$ replacement level was not significantly affected compared to the control FO diet (Ng et al., 2013). On the other hand, study on red sea bream, Pagrus major showed that the substitution of $\mathrm{PO}$ at $60 \%$ or higher affected the growth of the fish compared to control diet (Komilus et al., 2008) and substitution of palm oil at $69 \%$ also affected the growth of gilthead sea bream, Sparus aurata (Fountoulaki et al., 2009). These demonstrated that the potential use of PO products in aquaculture feed depends on the species and the level of oil used in the feed.

The present study showed that the growth performance and feed utilisation of hybrid grouper fed with COCO diet was comparable to the control diet FO although the FCR was slightly higher in fish fed with COCO. This is in agreement with previous study that the growth of fish was not affected by the $\mathrm{COCO}, \mathrm{CO}$, or squid oil. However, the feed efficiency and FCR were better in fish fed with $\mathrm{CO}$ compared to COCO and squid oil (Fitriyani et al., 2015). Although limited study can be found on the use of $\mathrm{COCO}$ in marine fish, $\mathrm{COCO}$ is commonly reported to be a good replacement for FO in freshwater fish (Bahurmiz and $\mathrm{Ng}$, 2007; Aderolu and Akinremi, 2009; Babalola and Adebayo, 2007; Apraku et al., 2017). Studies on African catfish, Clarias gariepinus showed that better growth performance was found in fish fed with COCO when compared with control that fed with FO (Bahurmiz and Ng, 2007; Aderolu and Akinremi, 2009; Babalola and Adebayo, 2007). Study in Nile tilapia, Oreochromis niloticus showed that $100 \%$ replacement of FO with COCO is possible as it resulted in better growth performance and FCR with no significant difference with the FO diet (Apraku et al., 2017).

The growth performance was lowest in hybrid grouper fed with $\mathrm{CO}$ diet, however, it was not significantly different with fish fed with $\mathrm{FO}$ diet. Similarly, in orange spotted grouper, E. coloides at 50\% replacement of FO, no significant difference in growth performance and survival was found between the fish fed with FO and CO treatments (Lin et al., 2007). Study on malabar grouper, E. malabaricus also resulted in no significant difference in growth and feed utilisation, and improvement of the non-specific immune system in fish fed with feed containing $50 \%$ of CO compared to FO-based diet (Lin and Shiau, 2003; 2007). In freshwater fish, study on gibel carp, Carassius auratus gibelio had showed that $\mathrm{CO}$ can be replaced up to $50 \%$ with anchovy oil in the diet without negative effects on the growth performance (Chen et al., 2011).Total replacement is possible in the red hybrid tilapia, Oreochromis sp. (Al-Souti et al., 2012) without any significant effects on the growth performance, FCR, and 
whole body proximate composition. The present study showed that $\mathrm{COCO}$ or $\mathrm{CO}$ can be an alternative source of lipid for hybrid grouper without compromising their growth.

The present study showed that the different VO influenced the whole body composition of the hybrid grouper. The whole body proximate composition showed that hybrid grouper contained higher body protein level in fish fed with $\mathrm{CPKO}, \mathrm{CO}$ and COCO compared to fish fed with FO, CPO and RBD POo. However, it showed contrasting result in the parental fish, brown-marbled grouper (Mohd Faudzi, 2013) and in humpback grouper (Shapawi et al., 2008) as the whole body composition was not influenced by the VO replacement. Similar result was reported in common carp, Cyprinus carpio L. that $50 \%$ replacement using $\mathrm{CO}$ resulted in higher protein content in fish body than fish fed with FO diet and $100 \%$ replacement level also showed similar result in tambaqui, Golossoma macropomum (Pereira et al., 2018). Similar result had been reported in different level of replacement of FO using palm oil (Komilus et al., 2008) and soyabean oil (Komilus et al., 2015) in Pagrus major.

In the present study, fish fed with $\mathrm{CPO}$ and RBD POo showed higher HSI value while CPKO and COCO showed significantly lower value $(\mathrm{P}<$ $0.05)$. Besides, a similar trend of the liver fat content with the HSI was also demonstrated significantly higher liver fat content was found in fish fed with CPO and RBD POo, and lowest in fish fed with CPKO and COCO. This suggests that the HSI in the present study is affected by the liver fat content among other factors. The experimental diets fatty acids profile showed that diets consisting of $\mathrm{CPO}$ and RBD POo contained high percentage of saturated palmitic (C16:0) and monounsaturated oleic acids (C18:1n-9) while CPKO and COCO showed high content of saturated fatty acid lauric (C12:0) and myristic acids (C14:0). The fatty acids may have influenced the deposition of fat in the liver. The present result is almost similar with the study on Japanese seabass that showed fish fed with diets added with C16:0, C18:0, C18:1n-9 or C18:3n-3 showed higher HSI and liver lipid content compared to fish fed with medium chain fatty acids C8:0 and C10:0 (Xu et al., 2016). In contrast with HSI and liver fat content, the VSI, whole body lipid content and muscle lipid content in the present study showed higher values in fish fed with $\mathrm{CO}$ (high level of C18:2n-6) and COCO (high level of $\mathrm{C} 12: 0$ and $\mathrm{C} 14: 0)$. This showed that diets containing $\mathrm{CPO}$ and RBD POo prompted higher lipid accumulation in the liver that led to higher $\mathrm{HSI}$, while diets containing $\mathrm{CO}$ and $\mathrm{COCO}$ induced higher values of VSI and muscle lipid deposition compared to other diets. This suggested oil with different fatty acid profile can affect the body indices, deposition of fat in the liver and muscle.
However, studies on other groupers showed that no significant difference on the HSI was reported for humpback grouper (HSI 1.18-1.73), C. altivelis (Shapawi et al., 2008), orange spotted grouper (HSI 1.6-2.2), E. coioides (Lin et al., 2007) when fed the fish with feed partially replaced with VO.

It is well documented that the dietary fatty acid composition can directly influence the fish body fatty acid composition (Izquierdo et al., 2003; Al-Souti et al., 2012; Komilus et al., 2015; Xu et al., 2016; MongeOrtiz et al., 2018) and similarly it was observed in the present study. At the end of the 12-week feeding trial, $50 \%$ of FO replacement with $\mathrm{VO}$ resulted in slightly lower level of n-3 PUFA and increased n-6 PUFA in fish muscle. Besides, considerable amount of DHA and EPA were accumulated in the muscle as shown in all the treatments. This is in agreement with a previous study which showed that malabar grouper, E. malabaricus was capable of directly incorporating dietary DHA and EPA into body tissue, in which diets low in DHA level tended to promote DHA deposition (Wu et al., 2002). Comparison between the fatty acid profile of muscle and liver, it demonstrated that the muscle contained relatively higher percentage of most of the PUFA such as C18:2n-6, C20:4n-6, EPA, DHA while the liver contained relatively higher percentage of the saturated and monounsaturated fatty acids. This demonstrated different utilisation or deposition of fatty acid between the two tissues and this is in agreement with previous studies (Izquierdo et al., 2003). Studies on P. major (Komilus et al., 2015) fed with different level of soyabean oil had resulted in high amount of EPA and DHA content in the muscle while high amount of total saturated fatty acid in the liver. Similar results was also found in greater amberjack, Seriola dumerili (Monge-Ortiz et al., 2018) fed with $50 \%$ and $100 \%$ replacement of fish oil using mixture of $(4: 1 \mathrm{v} / \mathrm{v}$, palm oil:linseed oil). Besides, Turchini et al. (2009) reviewed that when fish were fed with diet containing high concentration of saturated fatty acid it resulted in significantly less muscle deposition. The difference in the fat deposition of fish could be due to species specific differences. Further investigation on organ histology, hematology and sensory assessment should be carried out to determine the full potential of RBD POo as the preferred lipid sources in hybrid grouper juvenile.

\section{CONCLUSION}

Overall, VO tested in the present study have potential to be used as an alternative to partially replace FO in hybrid grouper feed. The present study showed that at 50\% replacement of FO with RBD POo is the most suitable VO for hybrid grouper as it promoted higher growth and feed utilisation. The different 
VO contributed to the differences in body condition, whole body composition, lipid deposition and fatty acid profile in the muscle and liver.

\section{ACKNOWLEDGEMENT}

The present study was partially funded by the Ministry of Higher Education, Malaysia under Niche Research Grant Scheme (NRGS0004). The authors would like to thank Sawit Kinabalu, Sabah for providing the various palm oils (CPO, RBD POo and $\mathrm{CPKO}$ ) throughout the feeding trial experiment.

\section{REFERENCES}

AOAC (1999). Official Methods of Analysis of the Association of Official Analytical Chemists. AOAC International, Washington, DC.

Aderolu, A Z and Akinremi, O A (2009). Dietary effects of coconut oil and peanut oil in improving biochemical characteristics of Clarias gariepinus juvenile. Turkish J. Fish Aquatic Science, 110: 9-15.

Al-Souti, A; Al-Sabahi, J; Soussi, B and Goddard, $S$ (2012). The effects of fish oil enriched diets on growth, feed conversion and fatty acid content of red hybrid tilapia, Oreochromis sp. Food Chemistry, 133: 723-727.

Apraku, A; Liu, L; Leng, X J; Rupia, E J and Ayisi, C L (2017). Evaluation of blended virgin coconut oil and fish oil on growth performance and resistance to Strptococcus iniae challenge of Nile tilapia Oreochromis niloticus. Egyptian J. Basic and Applied Sciences, 4: 175-184.

Arrokhman, S; Wijayanti, N and Soegianto, A (2017). Survival and osmoregulation of juvenile hybrid grouper (Epinephelus fuscoguttatus $\times$ Epinephelus lanceolatus) during acclimation in calciumsupplemented freshwater. Aquaculture International, 25: 693-704.

Babalola, T O and Adebayo, M A (2007). Effect of dietary lipid level on growth performance and feed utilization by Heteobranchus longifilis fingerlings. J. Fisheries International, 2 (1): 60-64.

Bahurmiz, O M and $\mathrm{Ng}, \mathrm{W}$ K (2007). Effects of dietary palm oil source on growth, tissue fatty acid composition and nutrient digestibility of red hybrid tilapia, Oreochromis sp., raised from stocking to marketable size. Aquaculture, 262: 382392.

Bunlipatanon, P and U-Taynapun, K (2017). Growth performance and disease resistance against
Vibrio vulnificus infection of novel hybrid grouper (Epinephelus lanceolatus $\times$ Epinephelus fuscoguttatus). Aquaculture Research, 48: 1711-1723.

Chen, J; Zhu, X; Han, D; Yang, Y; Lei, W and Xie, $S$ (2011). Effects of dietary n-3 HUFA on growth performance and tissue fatty acid composition of gibel carp Carassius auratus gibelio. Aquaculture Nutrition, 17: 476-485.

Chen, C Y; Chen, J S; Wang, S Q; Yu, C H and Li, Y Y (2017). Effects of different dietary ratios of linolenic to linoleic acids or docosahexaenoic to eicosapentaenoic acids on the growth and immune indices in grouper, Epinephelus coioides. Aquaculture, 473: 153-160.

Ch'ng, C L and Senoo, S (2008). Egg and larval development of a new hybrid grouper, tiger grouper Epinephelus fuscoguttatus $\times$ giant grouper $E$. lanceolatus. Aquaculture Science, 56: 505-512.

Christine, J V; Shamsudin, R and Baharuddin, A S (2014). Pre-treatment of oil palm fruits: A review. J. Food Engineering, 143: 123-131.

Food and Agriculture Organisation (FAO) (2009). The State of World Fisheries and Aquaculture: 2008. FAO, Rome, Italy.

Firdaus, R F; Lim, L S; Kawamura, G and Shapawi, R (2016). Assessment on the acceptability of hybrid grouper, Ephinephelus fuscoguttatus, \& $\mathrm{x}$ giant grouper E. lanceolatus, $\sigma^{1}$ to soybean meal-based diets. AACL Bioflux, 9: 284-290.

Fitriyani Kusdianto, H and Sukarti, K (2015). Effect of different dietary lipid sources on feed efficiency and feed conversion ratio of cantang grouper (Epinephelus sp.). Tropical Fishery Science J., 20: 8-14.

Folch, J; Lees, M and Stainley, G H S (1957). A simple method for isolation and purification of total lipid from animal tissue. J. Biology Chemistry, 226: 497-509.

Fountoulaki, E; Vasilaki, A; Hurtado, A; Grigorakis, K; Karacostas, I; Rigos, G; Kotzamanis, Y; Venou, B and Alexis, M N (2009). Fish oil substitution by vegetable oils in commercial diets for gilthead sea bream Sparus aurata L.; Effects on growth performance, flesh quality and fillet fatty acid profile: Recovery of fatty acid profiles by a fish oil finishing diet under fluctuating water temperatures. Aquaculture, 289: 317-326.

Glencross, B D; Hawkins, W E and Curnow, J G (2003). Restoration of the fatty acid composition of red seabream Pagrus auratus using a fish oil 
finishing diet after grow-out on plant oil based diets. Aquaculture Nutrition, 9: 406-418.

Hirazawa, N; Oshima, S; Hara, T; Mitsuboshi, T and Hata, K (2001). Antiparasitic effect of mediumchain fatty acids against the ciliate Cryptocaryon irritans infestation in the red sea bream Pagrus major. Aquaculture, 198: 219-228.

Ismail, R; Yong, A S K; Lim, L S; Kawamura, G and Shapawi, R (2018). Utilization of different dietary carbohydrate sources in hybrid grouper, Tiger grouper Ephinephelus fuscoguttatus, + x Giant grouper (E. lanceolatus, § juveniles. International J. Aquaculture Science, 9: 85-92.

Izquierdo, M S; Obach, A; Arantzamendi, L; Montero, D; Robaina, L and Roselund, G (2003). Dietary lipid sources for seabream and seabass; growth performance, tissue composition and flesh quality. Aquaculture Nutrition, 9: 397-407.

Izquierdo, M S; Montero, D; Robaina, L; Caballero, M J; Rosenlund, G and Gines, R (2005). Alterations in fillet fatty acid profile and flesh quality in gilthead seabream Sparus aurata fed vegetable oils for a long term period recovery of fatty acid profiles by fish oil feeding. Aquaculture, 250: 431-444.

Kamarudin, M S; Ramezani-Fard, E; Saad, S D and Harmin, S A (2012). Effects of dietary fish oil replacement by various vegetable oils on growth performance, body composition and fatty acid profile of juvenile Malaysian mahseer Tor tambroides. Aquaculture Nutrition, 18: 532-543.

Koh, I C C; Nurhamizah, B; Noor Dee'ana, Z and Sufian, M (2016). Effect of salinity on embryonic development and hatching of hybrid grouper, Epinephelus fuscoguttatus $\times$ Epinephelus lanceolatus. AACL Bioflux, 9: 1278-1285.

Komilus, C F; Shichi, N; Koshio, S; Ishikawa, M; Yoloyama, S; Michael, F R; Gao, J and Makita, C (2008). Influences of palm oil blended with fish oil on growth performances and lipid profiles of red sea bream Pagrus major. Aquaculture Science, 56: 317-326.

Komilus, C F; Koshio, S; Ishikawa, M; Yokoyama, S; Gao, J; Abdul Lader, M; Han, Y Z and Ren, T (2015). Influence of soya oil blend with fish oil on growth performance and lipid profile of red sea bream Pagrus major. J. Agrobiotech, 6: 15-31.

Lin, Y H and Shiau, S Y (2003). Dietary lipid requirement of grouper, Epinephelus malabaricus, and effects on immune responses. Aquaculture, 225: 243250.
Lin, Y H and Shiau, S Y (2007). Effects of dietary blend of fish oil with corn oil on growth and nonspecific immune responses of grouper, Epinephelus malabaricus. Aquaculture Nutrtition, 13: 137-144.

Lin, H Z; Liu Y J; He, J G; Zheng, W H and Tian, L Z (2007). Alternative vegetable lipid sources in diets for grouper, Epinephelus coioides (Hamilton): Effects on growth, and muscle and liver fatty acid composition. Aquaculture Research, 38: 1605-1611.

Mohd Faudzi, N (2013). Fish Oil Replacement with Vegetable Oils Using Different Sources of Fish Meal in Pelleted Feeds for Juvenile Tiger Grouper, Epinephelus fuscoguttatus. Master's thesis. Universiti Malaysia Sabah, Malaysia.

Mohd Faudzi, N; Yong, A S K; Shapawi, R; Senoo, S; Biswas, A and Takii, K (2017). Soy protein concentrate as an alternative in replacement of fish meal in the feeds of hybrid grouper, brown-marbled grouper (Epinephelus fuscoguttatus) x giant grouper (E.lanceolatus) juvenile. Aquaculture Research, 49(1): $1-11$.

MPOC (2017). Annual Report 2017. Malaysian Palm Oil Council, Malaysia.

Monge-Ortiz, R; Tomás-Vidal, A; RodriguezBarreto, D; Martínez-Llorens, S; Péres, J A; JoverCerdá, M and Lorenzo, A (2018). Replacement of fish oil with vegetable oil blends in feeds for greater amberjack Seriola dumrili juveniles: Effect on growth performance, feed efficiency, tissue fatty acid composition and flesh nutritional value. Aquaculture Nutrition, 24: 605-615.

Mourente, G and Bell, J G (2006). Partial replacement of dietary fish oil with blends of vegetable oils (rapeseed, linseed and palm oils) in diets for European sea bass Dicentrarchus labrax L. over a long term growth study: Effects on muscle and liver fatty acid composition and effectiveness of a fish oil finishing diet. Comparative Biochemistry and Physiology Part B: Biochemistry and Molecular Biology, 145: 389-399.

Mustafa, S; Senoo, S and Luin, M (2013). Response of pure stock of coral reef tiger grouper and hybrid grouper to simulated ocean acidification. International J. Climate Change: Impact and Responses, 5: $47-54$

Nasopoulu, C and Zabetakis, I (2012). Benefits of fish oil replacement by plant originated oils in compounded fish feeds. A review. Food Science Technology, 47: 217-244.

Ng, W K; Chong, C H; Wang, Y and Romano, N (2013). Effects of dietary fish and vegetable oils on 
the growth, tissue fatty acid composition, oxidative stability and vitamin E content of red hybrid tilapia and efficacy of using fish oil finishing diets. Aquaculture, 372: 97-110.

Oil World (2017). Oil World Annual 2017. ISTA MIELKE GMBH, Hamburg, Germany.

Olsen, R E; Waagbø, R; Melle, W; Ringø, E and Lall, S P (2011). Alternative Marine Resources. Fish Oil Replacement and Alternative Lipid Sources in Aquaculture Feeds (Turchini, G M; Ng, W K and Tocher, D R eds.). Taylor \& Francis, CRC Press, Boca Raton. p. 237-324.

Othman, A R; Kawamura, G; Senoo, S and Fui, C F (2015). Effects of different salinities on growth, feeding performance and plasma cortisol level in hybrid TGGG (tiger grouper, Epinephelus fuscoguttatus $\times$ giant grouper, Epinephelus lanceolatus) juveniles. International Research J. Biological Sciences, 4: $15-20$.

Pereira, T R; Paulino, R R; Almeida, C A L; Rosa, P V; Orlando, T M and Fortes-Silva, R (2018). Oil sources administered to tambaqui, Colossoma macropum: Growth, body composition and effect of masking organoleptic properties and fasting on diet preference. Applied Animal Behaviour Science, 199: 103-110.

Piedecausa, M A; Mazón, M J; García, B and Hernández, M D (2007). Effects of total replacement of fish oil by vegetable oils in sharpsnout seabream Diplodus puntazzo diets. Aquaculture, 263: 211-219.

Røsjø, C; Nordrum, S; Olli, J J; Krogdahl, A; Ruyter, B and Holm, H (2000). Lipid digestibility and metabolism in Atlantic salmon Salmo salar fed medium-chain triglycerides. Aquaculture, 190: 65-76.

Shapawi, R; Mustafa, S and Ng, W K (2008). Effects of dietary fish oil replacement with vegetable oils on growth and tissue fatty acid composition of humpback grouper, Cromileptes altivelis (Valenciennes). Aquacultre Resarch, 39: 315-32.

Torstensen, B E; Froyland, L and Lie, O (2004). Replacing dietary fish oil with increasing levels of rapeseed oil and olive oil effects on Atlantic salmon Salmo salar L. tissue and lipoprotein lipid composition and lipogenic enzyme activities. Aquaculture Nutrition, 10: 175-192.

Turchini, G M; Torstensen, B E and Ng, W K (2009). Fish oil replacement in finfish nutrition-review. Aquaculture, 1: 10-57.

Turchini, G M; Ng, W K and Tocher, D R (2010). Fish Oil Replacement and Alternative Lipid Sources in Aquaculture Feeds. Boca Raton: CRC Press.

USDA (2017). Oilseeds: Worlds Markets and Trade, 2017. United States Department of Agriculture, Foreign Agricultural Service.

Wan Ahmad, W A R; Stone, D A J and Schuller, K A (2013). Dietary fish oil replacement with palm or poultry oil increases fillet oxidative stability and decreases liver glutathione peroxidise activity in barramundi Lates calcarifer. Fish Physiology Biochemistry, 39: 1631-1640.

Wu, F C L; Ting, Y Y and Chen, H Y (2002). Docosahexaenoic acid is superior to eicosapentaenoic acid as essential fatty acid for growth of grouper, Epinephelus malabaricus. The J. Nutrition, 132: 72-79.

Xu, H; Dong, X; Zuo, R; Mai, K and Ai, Q (2016). Response of juvenile Japanese seabass, Lateolabrax japonicus to different dietary fatty acid profiles: Growth performance, tissue lipid accumulation, liver histology and flesh quality. Aquaculture, 461: 40-47.

Yoshikara, R and Satoh, M (1989). Chemistry for Fisheries (in Japanese). Kouseiya-kouseikalu, Tokyo, Japan. p. 77-78. 